\title{
Identification of a Cranberry Juice Product that Inhibits Enteric CYP3A-Mediated First-Pass Metabolism in Humans
}

\author{
Ngoc Ngo, ${ }^{1}$ Zhixia Yan, Tyler N. Graf, Daniel R. Carrizosa, Angela D. M. Kashuba, \\ E. Claire Dees, Nicholas H. Oberlies, and Mary F. Paine \\ Division of Pharmacotherapy and Experimental Therapeutics, Eshelman School of Pharmacy (N.N., Z.Y., A.D.M.K., M.F.P.) and \\ Division of Hematology and Oncology, School of Medicine (D.R.C., E.C.D.), University of North Carolina, Chapel Hill, North \\ Carolina; and Natural Products Laboratory, Research Triangle Institute, Research Triangle Park, North Carolina (T.N.G., N.H.O.)
}

Received September 29, 2008; accepted December 23, 2008

\begin{abstract}
:
An in vivo study in rats showed a cranberry juice product to inhibit the intestinal first-pass metabolism of the CYP3A substrate nifedipine. However, a clinical study involving the CYP3A probe substrate midazolam and a different cranberry juice product showed no interaction. Because the composition of bioactive components in natural products can vary substantially, a systematic in vitro-in vivo approach was taken to identify a cranberry juice capable of inhibiting enteric CYP3A in humans. First, the effects of five cranberry juices, coded A through $E$, were evaluated on midazolam 1 '-hydroxylation activity in human intestinal microsomes. Juice $E$ was the most potent, ablating activity at $0.5 \%$ juice $(\mathrm{v} / \mathrm{v})$ relative to control. Second, juice E was fractionated to generate hexane-, chloroform-, butanol-, and aqueous-soluble fractions. The hexaneand chloroform-soluble fractions at $50 \mu \mathrm{g} / \mathrm{ml}$ were the most po-
\end{abstract}

tent, inhibiting by 77 and $63 \%$, respectively, suggesting that the CYP3A inhibitors reside largely in these more lipophilic fractions. Finally, juice $E$ was evaluated on the oral pharmacokinetics of midazolam in 16 healthy volunteers. Relative to water, juice E significantly increased the geometric mean area under the curve $(\mathrm{AUC})_{0-\infty}$ of midazolam by $\sim 30 \%(p=0.001)$, decreased the geometric mean 1'-hydroxymidazolam/midazolam $\mathrm{AUC}_{0-\infty}$ ratio by $\sim 40 \%(p<0.001)$, and had no effect on geometric mean terminal half-life, indicating inhibition of enteric, but not hepatic, CYP3Amediated first-pass metabolism of midazolam. This approach both showed a potential drug interaction liability with cranberry juice and substantiated that rigorous in vitro characterization of dietary substances is required before initiation of clinical drug-diet interaction studies.
Cranberry juice has become popular as a natural alternative for the prevention of urinary tract infections (UTIs), which have a high incidence in women and elderly persons (Foxman, 2003; McMurdo et

This work was supported in part by the National Institutes of Health National Institute of General Medical Sciences [Grant R01-GM077482]; the National Institutes of Health National Center for Research Resources [Grant M01-RR000046]; the University of North Carolina Research Council; and the University of Washington School of Pharmacy Elmer M. Plein Endowed Research Fund.

Parts of this work were previously presented as follows: Ngo M, Dees EC, and Paine MF (2007) Cranberry juice delays midazolam absorption in healthy volunteers. American Society for Clinical Pharmacology and Therapeutics Meeting; 2007 Mar 21-24; Anaheim, CA; American Society for Clinical Pharmacology and Therapeutics, Alexandria, VA; Ngo N, Yan Z, Graf TN, Carrizosa DR, Dees EC, Oberlies NH, and Paine MF (2008) Identification of a cranberry juice product capable of inhibiting enteric CYP3A-mediated first-pass metabolism in humans. 7th Annual Oxford International Conference on the Science of Botanicals and the American Society of Pharmacognosy 4th Interim Meeting; 2008 Apr 12-16; Oxford, MS. National Center for Natural Products Research, Oxford, MS.

${ }^{1}$ Current affiliation: Clinical Pharmacology, Quintiles Transnational, Overland Park, Kansas.

Article, publication date, and citation information can be found at http://dmd.aspetjournals.org.

doi:10.1124/dmd.108.024968. al., 2005; Jepson and Craig, 2008). Moreover, cranberry juice has shown efficacy in reducing UTIs in women with recurrent episodes and in reducing bacteriuria in elderly persons (Avorn et al., 1994; Stothers, 2002). Clinical benefits are associated with chronic use (months) and with a regular frequency of consumption (daily) (Jepson and Craig, 2008). Studies have shown that the prophylactic nature of cranberry juice is a result of inhibition of the adhesion of bacterial fimbriae to uroepithelial cells, rather than because of urinary acidification (Liu et al., 2006; Gupta et al., 2007). Cranberry juice also has shown a beneficial effect against drug-resistant bacteria (Howell and Foxman, 2002), which could be important in institutional settings, where nosocomial infections are frequent.

Despite these seemingly beneficial attributes, studies have suggested that cranberry juice may be capable of eliciting clinically relevant interactions with certain medications, albeit the literature is inconsistent. For example, in rats, cranberry juice was as effective as grapefruit juice in enhancing the systemic exposure of the calcium channel antagonist and cytochrome P450 3A (CYP3A) substrate nifedipine (Uesawa and Mohri, 2006). Compared with saline, both grapefruit juice and cranberry juice significantly increased the area under the curve (AUC) of nifedipine by $60 \%$. Moreover, similar to grapefruit juice, cranberry juice seemed to inhibit enteric, but not hepatic, CYP3A-mediated first-pass metab- 
olism, as exemplified by the lack of effect on drug systemic half-life. In contrast, a human study involving the CYP3A substrate cyclosporine and cranberry juice indicated no interaction (Grenier et al., 2006). However, cyclosporine is also a substrate for the enteric efflux transporter P-glycoprotein (P-gp), which complicates the interpretation of whether cranberry juice inhibits enteric CYP3A. In addition, only a single glass $(240 \mathrm{ml})$ of juice was given, which conflicts with the general recommendation of consuming several glasses daily for the prevention of UTIs (Lynch, 2004). Most recently, Lilja et al. (2007) reported that cranberry juice, taken thrice daily for 10 days, had no effect on the pharmacokinetics of the CYP3A probe substrate midazolam, which was administered as a single oral dose on day 5 . Although a more appropriate probe substrate and real-life scenario of long-term exposure was examined, some comments warrant mention. In particular, no rationale was provided as to why cranberry juice was taken for 10 days, why midazolam was given on day 5, or why a sample size of 10 was chosen. Nevertheless, the current literature suggests that cranberry juice has a drug interaction liability for rats, but not humans, and thus has no clinical concerns.

An oversight to the aforementioned conclusion is that each study examined a single brand of juice, which, like all products derived from natural substances, vary considerably in the composition of bioactive ingredients (Paine and Oberlies, 2007). Accordingly, an alternate conclusion is that the product tested in the rat study contained a suite of CYP3A inhibitory compounds at an aggregate concentration sufficient to elicit an inhibitory effect in vivo, whereas the products used in the clinical studies did not. However, this supposition is not easily assessable because none of the authors reported an in vitro characterization of the test juice with respect to enteric CYP3A inhibitory activity before initiation of the in vivo study. Because such a priori testing is required for evaluation of the metabolic consequence of new chemical entities, it follows that similar procedures should be implemented for evaluation of the effects of dietary substances on drug disposition (Paine and Oberlies, 2007).

Based on the inconsistencies in the literature regarding a potential cranberry juice effect, along with the challenges that present when evaluating the drug interaction liability of a dietary substance, cranberry juice was selected as a model substance to test the hypothesis that a systematic approach should be used to identify clinically relevant drug-diet interactions prospectively. First, multiple cranberry juice products were tested as inhibitors of enteric CYP3A activity using midazolam and human intestinal microsomes. Second, the most potent juice was fractionated using established natural products chemistry techniques, and the resultant fractions were tested as inhibitors of enteric CYP3A activity. Third, the physicochemical effects of the selected test juice on the absorption of midazolam were evaluated using the human intestinal cell line Caco-2. Finally, the test juice was evaluated on the oral pharmacokinetics of midazolam in healthy volunteers. This approach identified a cranberry juice that elicited a pharmacokinetic interaction with an established CYP3A probe substrate, substantiating that a rigorous in vitro characterization of a dietary substance should be undertaken before initiation of a clinical drug-diet interaction study. These observations also raise concerns for individuals taking certain medications concomitantly with certain brands of cranberry juice.

\section{Materials and Methods}

Materials and Chemicals. Human intestinal microsomes (HIMs) were prepared previously from mucosal scrapings obtained from the jejunal portion of a donor small intestine that was shown to be devoid of readily detectable CYP3A5 immunoreactive protein using a selective anti-CYP3A5 antibody (Paine et al., 2006). Baculovirus-insect cell-expressed CYP3A4 and CYP3A5 (coexpressed with cytochrome P450 reductase but not supplemented with cytochrome $b_{5}$ ) and 1 '-hydroxymidazolam (used for analysis of the in vitro samples) were purchased from BD Gentest (Woburn, MA). Midazolam maleate, alprazolam, ketoconazole, NADPH, ammonium hydroxide (28\% in water), and formic acid were purchased from Sigma-Aldrich (St. Louis, MO). BioCoat culture inserts $\left(4.2 \mathrm{~cm}^{2}\right.$; BD Biosciences, San Jose, CA), murine laminin, Dulbecco's modified Eagle's medium, nonessential amino acids, vitamin E, gentamicin, sodium selenite, and zinc sulfate were purchased from sources as described previously (Mouly et al., 2004). Fetal bovine serum was purchased from Lonza Walkersville Inc. (Walkersville, MD). Disposable syringe filters (Uniflo25, pore size $0.2 \mu \mathrm{m}$ ) were purchased from Whatman Schleicher and Schuell (Keene, NH). 1'-Hydroxymidazolam and $\mathrm{d}_{4}-1^{\prime}$-hydroxymidazolam (used for the analysis of human plasma) were purchased from Cerilliant (Round Rock, TX). Methanol, hexane, chloroform, and acetonitrile were high-performance liquid chromatography (HPLC) grade (Thermo Fisher Scientific, Waltham, MA); 1-butanol was aqueous counting scintillant reagent grade (Mallinckrodt Baker, Inc., Phillipsburg, NJ).

Natural Study Materials and Extraction/Fractionation of Cranberry Juice from Vendor E. The following bottled, nonfrozen juices, which contained cranberry juice in some form, were purchased from a local grocery store: Lakewood Organic Fresh Pressed Blend Cranberry (Lakewood, Miami, FL; Lot 147H), Ocean Spray Cranberry Juice Cocktail (Ocean Spray Cranberries, Inc., Lakeville-Middleboro, MA; Lot B0513060220), Market Pantry Juice Cocktail Cranberry (Target Corp., Minneapolis, MN: Lot 11F 13:11), 365 Brand 100\% Cranberry Juice (Whole Foods Market, Austin, TX; Lot 02/17/ 08), and R.W. Knudsen Family Cranberry Juice Concentrate (Knudsen and Sons, Inc., Orville, $\mathrm{OH}$ ). As described in the labeling, the Lakewood brand contained fresh pressed juice and puree from whole ripe certified organic apples, organic cranberries, and organic grapes; the Ocean Spray brand contained filtered water, $27 \%$ cranberry juice (from concentrate and cranberry juice), high fructose corn syrup, and ascorbic acid; the Market Pantry brand contained filtered water, high fructose corn syrup, $27 \%$ cranberry juice (from concentrate), and ascorbic acid; the 365 brand contained filtered water, cranberry juice, and cranberry juice concentrate; and the Knudsen brand was a cranberry juice concentrate that contained no added sugars or other fruit juices. To avoid any perceived conflicts of interest, the identities of the juices were coded as vendors A, B, C, D, and E.

The juice from vendor E was extracted and fractionated using natural products chemistry techniques, combining methods that were developed for processing liter-scale bacterial cultures (Cain et al., 2003) with those that were optimized for dried plant materials (Kinghorn et al., 2003; Phifer et al., 2007) (Fig. 1). In brief, 1.6 liters of juice were freeze-dried; the resultant powder was sonicated in methanol; and the entire suspension was stirred overnight. Solids were removed via filtration, and the eluent was reduced in vacuo to yield a methanol extract. This extract was partitioned between 9:1:10 methanol/water/ hexane, yielding a hexane fraction and an aqueous methanol fraction. The latter was partitioned subsequently between $4: 1: 5$ chloroform/methanol/water. The lower organic layer was removed and dried in vacuo to yield the chloroform-soluble fraction. The upper aqueous layer was shaken with 1-butanol to yield a 1-butanol fraction and an aqueous fraction. Each of the four resultant fractions (hexane-, chloroform-, butanol-, and aqueous-soluble) was dried in vacuo and stored at $-80^{\circ} \mathrm{C}$ before in vitro testing.

In Vitro Characterization of Commercially Available Cranberry Juices. Testing of whole cranberry juices as inhibitors of enteric CYP3A4 activity. The inhibitory effects of the five cranberry juice products on midazolam 1'-hydroxylation were evaluated using CYP3A5-negative HIMs. Midazolam and ketoconazole were dissolved in methanol to yield stock solutions of 3 and $2 \mathrm{mM}$, respectively. These stock solutions were further diluted in water and methanol, respectively, to yield working concentrations of 0.3 and 0.2 $\mathrm{mM}$. Each cranberry juice was diluted in water to yield working concentrations of 1 and $10 \%(\mathrm{v} / \mathrm{v})$. NADPH was prepared fresh in potassium phosphate buffer $(0.1 \mathrm{M}, \mathrm{pH} 7.4)$ to yield a working concentration of $10 \mathrm{mM}$. Incubation mixtures consisted of HIMs $(0.05 \mathrm{mg} / \mathrm{ml}$ microsomal protein), midazolam (3 $\mu \mathrm{M})$, cranberry juice $(0.05$ or $0.5 \%)$, and potassium phosphate buffer $(0.1 \mathrm{M}$, $\mathrm{pH}$ 7.4); control incubation mixtures contained water in place of cranberry juice. As a positive control for CYP3A inhibition, incubation mixtures were prepared that contained ketoconazole $(2 \mu \mathrm{M})$ in place of cranberry juice; control incubation mixtures contained $1 \%$ methanol in place of ketoconazole. 

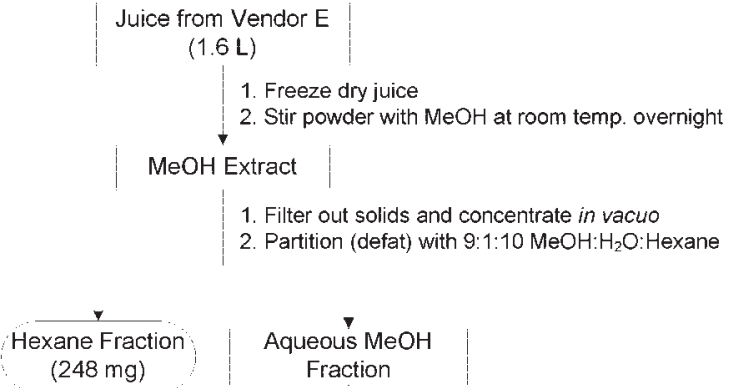

$$
\text { Aqueous } \mathrm{MeOH}
$$$$
\text { Fraction }
$$

1. Concentrate in vacuo

2. Partition between $4: 1: 5 \mathrm{CHCl}_{3}: \mathrm{MeOH}: \mathrm{H}_{2} \mathrm{O}$
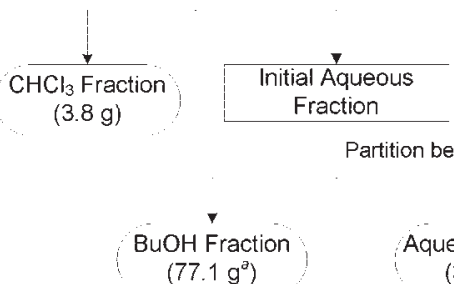

Partition between $1: 1 \mathrm{n}-\mathrm{BuOH}: \mathrm{H}_{2} \mathrm{O}$

$\left(77.1 \mathrm{~g}^{2}\right)$
Aqueous Fraction

$\left(322.5 \mathrm{~g}^{2}\right)$
FIG. 1. Extraction and fractionation scheme used for the processing of cranberry juice from vendor E. ${ }^{a}$ The weights for the butanol- and aqueous-soluble fractions are approximate. Because of the large size, it is difficult to remove all the residual solvent, which could raise the measured weights slightly.

All the incubation mixtures were equilibrated in a shaking water bath at $37^{\circ} \mathrm{C}$ for $5 \mathrm{~min}$ before initiating the reactions with NADPH ( $1 \mathrm{mM}$ final concentration) to yield a final volume of $200 \mu \mathrm{l}$. After $4 \mathrm{~min}, 100-\mu \mathrm{l}$ aliquots were removed and mixed with $200 \mu \mathrm{l}$ of acetonitrile containing $0.02 \mu \mathrm{g} / \mathrm{ml}$ internal standard (alprazolam). After centrifugation $\left(1450 \mathrm{~g}\right.$ for $10 \mathrm{~min}$ at $\left.4^{\circ} \mathrm{C}\right)$, the supernatants were analyzed for 1'-hydroxymidazolam by HPLC/tandem mass spectrometry (MS/MS) as described previously (Wang et al., 2007). The amount of $1^{\prime}$-hydroxymidazolam formed was linear with respect to incubation time and mass of microsomal protein.

Testing of fractions prepared from cranberry juice $E$ on CYP3A activity. Each of the four fractions prepared from cranberry juice $\mathrm{E}$ was dissolved in methanol to yield working concentrations of 1 and $5 \mathrm{mg} / \mathrm{ml}$. Incubation mixtures were prepared in a similar manner as described in the preceding section, only the whole juice was replaced with juice fraction (10 or $50 \mu \mathrm{g} / \mathrm{ml})$, and control incubation mixtures contained $1 \%$ methanol in place of juice fraction. The inhibitory effects of cranberry juice $\mathrm{E}$ and corresponding fractions were also tested with recombinant CYP3A4 and CYP3A5 (rCYP3A4 and rCYP3A5, respectively). Incubation mixtures were prepared in a similar manner, only the HIMs were replaced with $\mathrm{rCYP} 3 \mathrm{~A}(10 \mathrm{pmol} / \mathrm{ml})$. The reaction mixtures were further processed and analyzed for 1'-hydroxymidazolam as described in the preceding section. The amount of $1^{\prime}$-hydroxymidazolam formed was linear with respect to incubation time and amount of rCYP3A enzyme.

Evaluation of potential physicochemical effects of cranberry juice $E$ on midazolam absorptive permeability in Caco-2 cell monolayers. The Caco-2 cell clone P27.7 (Schmiedlin-Ren et al., 1997), passage 24, was seeded onto laminin-coated BioCoat culture inserts $\left(4.2 \mathrm{~cm}^{2} /\right.$ insert; BD Biosciences) at a density of $\sim 4 \times 10^{5}$ cells $/ \mathrm{cm}^{2}$ as described previously (Mouly et al., 2004). Cell cultures were maintained in complete growth medium (Mouly et al., 2004) until reaching confluence, when transepithelial electrical resistance (TEER) was $\geq 250 \Omega-\mathrm{cm}^{2}$. On reaching confluence ( $\sim 5$ days postseeding), the cells were maintained in differentiation medium (Mouly et al., 2004) for 3 weeks. Throughout the experimental period, cell cultures were maintained in a humidified incubator at $37^{\circ} \mathrm{C}$ and $5 \%$ carbon dioxide air atmosphere.

Plain incubation medium (differentiation medium devoid of fetal bovine serum) served as the control solution. The high sugar content solution was prepared by dissolving common table sugar (the same as that used in the clinical study) in incubation medium at a concentration of $50 \mathrm{~g} / \mathrm{l}$. The low $\mathrm{pH}$ solution was prepared by adjusting the incubation medium $\mathrm{pH}$ (7.4) to 5.5 with $5 \mathrm{~N}$ hydrochloric acid. Both the high sugar and low $\mathrm{pH}$ solutions were sterile-filtered using $0.2-\mu \mathrm{m}$ disposable syringe filters. The cranberry juice solution was prepared on the day of the experiment by diluting juice $\mathrm{E}$ with incubation medium to yield a $25 \%$ juice (1 part concentrate/12 parts water, according to the manufacturer's labeling) and adjusting the $\mathrm{pH}$ from 2.5 to 7.4 with $1 \mathrm{~N}$ sodium hydroxide. Because the molecular mass(es) of the potential CYP3A inhibitor(s) in the selected juice was not known, the $25 \%$ juice was not sterile-filtered so as to retain all the active and inactive ingredients.

The control and three treatment solutions were spiked with midazolam to yield a final concentration of $3 \mu \mathrm{M}(0.1 \% \mathrm{v} / \mathrm{v}$ methanol). Control or treatment solution $(1.5 \mathrm{ml})$ was added to the apical side, and plain incubation medium $(1.5 \mathrm{ml})$ was added to the basolateral side of triplicate culture inserts. After 0 , $0.25,0.5,1,2,3$, and $4 \mathrm{~h}$ at $37^{\circ} \mathrm{C}$, a $20-\mu \mathrm{l}$ aliquot was collected from the apical and basolateral compartment of each insert and transferred to the wells of a 96-well plate, each of which contained $80 \mu \mathrm{l}$ of water, followed by $200 \mu \mathrm{l}$ of acetonitrile. TEER was measured at each time point to assess monolayer integrity. After the 4-h collection, the remaining apical and basolateral media were aspirated, and the remaining cells were collected by scraping into 400 $\mu \mathrm{l}$ of cold incubation medium, followed by $1.2 \mathrm{ml}$ of acetonitrile. All the apical, basolateral, and cell collections were stored at $-80^{\circ} \mathrm{C}$ pending analysis for midazolam by HPLC/MS/MS (Wang et al., 2007) using the characteristic single reaction monitoring transition, $\mathrm{m} / \mathrm{z}, 326.3 \rightarrow 291.1$. Calibration standards were prepared in Dulbecco's modified Eagle's medium and ranged from 15 to $5000 \mathrm{nM}$.

The apparent permeability coefficient $\left(\mathrm{P}_{\mathrm{app}}\right)$ of midazolam in the apical to basolateral direction was calculated according to the following equation: $\mathrm{P}_{\text {app }}=(\mathrm{dQ} / \mathrm{dt}) \times\left(1 / \mathrm{AC}_{0}\right)$, where $d Q / d t$ denotes the rate of midazolam translocation from the apical to the basolateral compartment under sink conditions (i.e., before $>10 \%$ of the initial dose translocated to the basolateral compartment), $A$ denotes the surface area of the culture insert $\left(4.2 \mathrm{~cm}^{2}\right)$, and $C_{O}$ denotes the concentration of midazolam in the apical compartment at time 0 .

Human Volunteer Study. Clinical protocol and study subjects. Both the University of North Carolina Office of Human Research Ethics/Biomedical Institutional Review Board and Clinical Research Advisory Committee reviewed and approved the study protocol. Written informed consent and Health Insurance Portability and Accountability Act authorization were obtained from each volunteer before participation. Healthy men and nonpregnant women (eight each) were enrolled, excluding one man and one woman who withdrew during the first phase because of a scheduling conflict and discomfort from venipuncture, respectively. The men ranged in age from 20 to 58 years and the women from 24 to 52 years. The participants were self-identified as white (four men, six women), African American (three men, one woman), Hispanic (one man), or Asian (one woman). Before enrollment, each participant underwent a medical history, physical exam, liver function tests, and complete blood count. Each woman also underwent a serum pregnancy test. None of the participants were taking known modulators of CYP3A activity, except for two women (one white, one African American) who were taking oral contraceptives for at least 5 to 7 years and assumed to be at steady state. Other concomitant medications included levothyroxine (one white woman), hydrochlorothiazide (one white woman), and multivitamin (one white woman and one African American woman).

Preparation of double-strength cranberry juice. The selected cranberry juice (E) was reconstituted with half the recommended volume of water to yield a 200\% concentrated juice. To minimize the variation in ingredients, multiple bottles of juice from the same lot were combined to yield a single stock concentrate. Common table sugar (Dixie Crystals, Port Wentworth, GA) was added ( $5 \mathrm{~g} / \mathrm{oz}$, or $166 \mathrm{~g} / \mathrm{l})$ to improve palatability.

Study design. A prospective randomized, crossover, open-label study was conducted at the University of North Carolina General Clinical Research Center. The participants were asked to abstain from all fruit juices 1 week before and during the study and from alcohol and caffeinated beverages the evening before each study day. Participants were admitted to the General Clinical Research Center the evening before each of two study phases, which were separated by at least a 2-week interval. All the women underwent a repeat serum pregnancy test on admission for each phase. Vital signs (blood pressure, temperature, pulse, respirations) and oxygen saturation were obtained on admission and monitored periodically throughout each phase. After an overnight fast, each participant was given three 240-ml glasses of water or doublestrength cranberry juice, each separated by a 15 -min interval. The participant was given $5 \mathrm{mg}$ of midazolam syrup (Roxane Laboratories, Inc., Columbus, 
$\mathrm{OH})$ with the third glass. Blood $(5 \mathrm{ml})$ was collected by venous puncture from an indwelling catheter before dosing and at $0.25,0.5,1,1.5,2,3,4,6,8$, and $12 \mathrm{~h}$ postdose. Blood was centrifuged within $1 \mathrm{~h}$ of collection; plasma was saved and stored at $-20^{\circ} \mathrm{C}$ pending analysis for midazolam and $1^{\prime}$-hydroxymidazolam. Stability studies have shown that both analytes are stable in human plasma at $-20^{\circ} \mathrm{C}$ for 6 months, varying at most by $15 \%$ of nominal (A.D.M. Kashuba, unpublished observations). Accordingly, all the plasma samples were analyzed within 6 months of collection.

Analysis of Human Plasma for Midazolam and 1'-Hydroxymidazolam. Midazolam. Concentrations of midazolam were determined using a modified, validated reverse-phase HPLC method with mass spectrometric detection (Kanazawa et al., 2004). In brief, $300 \mu \mathrm{l}$ of plasma was added to $100 \mu \mathrm{l}$ of methanol containing $10 \mathrm{ng} / \mathrm{ml}$ alprazolam (internal standard). The diluted plasma was extracted with $1.5 \mathrm{ml}$ of $t$-butyl ether, evaporated to dryness using a TurboVap LV Evaporator (Caliper Life Sciences, Hopkinton, MA) at $30^{\circ} \mathrm{C}$ for $10 \mathrm{~min}$, and reconstituted with $100 \mu \mathrm{l}$ of an acetonitrile/water/acetic acid mixture before injection onto an Agilent Technologies (Santa Clara, CA) 1100 LC-MSD system. Analytes were separated using a Luna C8 column $(3.0 \mu \mathrm{m}$, $2 \times 100 \mathrm{~mm}$; Phenomenex, Torrance, CA) and a gradient elution with a flow rate of $200 \mu \mathrm{l} / \mathrm{min}$. Calibration curves ranged from 0.1 to $500 \mathrm{ng} / \mathrm{ml}$. Interday and intraday coefficients of variation across the range of concentrations were less than $8 \%$.

$1^{\prime}$-Hydroxymidazolam. Plasma $(0.5 \mathrm{ml})$ was transferred to $1.5-\mathrm{ml}$ amber tubes and diluted with $0.5 \mathrm{ml}$ of Dulbecco's phosphate-buffered saline, after which $8.5 \mu \mathrm{l}$ of the internal standard, $\mathrm{d}_{4}-1^{\prime}$-hydroxymidazolam $(1.0 \mu \mathrm{g} / \mathrm{ml})$, was added. Solid-phase extraction (SPE) was carried out using Strata-X reverse-phase polymeric cartridges ( $33 \mu \mathrm{m}, 60 \mathrm{mg}, 3 \mathrm{ml}$; Phenomenex). SPE cartridges were conditioned with $2 \mathrm{ml}$ of methanol, equilibrated with $3 \mathrm{ml}$ of water, and loaded with $1 \mathrm{ml}$ of the diluted plasma. The SPE cartridges were washed, successively, with $2 \mathrm{ml}$ of water, $2 \mathrm{ml}$ of water $/ 0.1 \%$ ammonium hydroxide, and twice with $2 \mathrm{ml}$ of 95:5:0.1 water/acetonitrile/ammonium hydroxide. The washed columns were dried under suction for $1 \mathrm{~min}$ and eluted into amber tubes with $1.2 \mathrm{ml}$ of acetonitrile $/ 0.1 \%$ ammonium hydroxide. All the tubes were dried partially under a nitrogen atmosphere overnight (to $\sim 400$ $\mu \mathrm{l})$ and then dried to completion in a Speedvac (SVC-200H; Thermo Fisher Scientific). All the SPE purification steps were performed under low light conditions. Residues were resuspended in $400 \mu 1$ of 90:10:0.1 water/acetonitrile/ammonium hydroxide for analysis by HPLC/MS/MS. Calibration standards and quality controls were prepared in blank human plasma (SeraCare Life Sciences, Inc., Milford, MA) and purified by SPE following the same procedures. Calibration curves ranged from 0.05 to $120 \mathrm{ng} / \mathrm{ml}$. Quality controls were 0.35 and $17 \mathrm{ng} / \mathrm{ml}$.

HPLC/MS/MS analysis was performed using a PE-Sciex API-365 triple quadrupole mass spectrometer (Applied Biosystems, Foster City, CA) coupled with a PerkinElmer Life and Analytical Sciences (Waltham, MA) 200 series micro-HPLC system. Liquid chromatography was carried out via $100-\mu 1$ injections with a Luna C18(2) column $(3 \mu \mathrm{m}, 50 \times 2.0 \mathrm{~mm}$; Phenomenex $)$ maintained at $30^{\circ} \mathrm{C}$. The mobile phase, at a flow rate of $300 \mu \mathrm{l} / \mathrm{min}$, consisted of water (solvent A) and acetonitrile (solvent $\mathrm{B}$ ), both modified with $0.1 \%$ ammonium hydroxide $(\mathrm{v} / \mathrm{v})$. A linear gradient was used that changed the A/B solvent ratio from 95:5 to 5:95 over $4 \mathrm{~min}$, before re-equilibration to the initial conditions for $6 \mathrm{~min}$. A diverter valve redirected the column effluent to waste during the initial and later part of the gradient, during times when the compounds of interest did not elute. General MS conditions were as follows: source, Turbo Ionspray (electrospray ionization); ion polarity, positive; spray voltage, $5000 \mathrm{~V}$; source gas, nitrogen; nebulizer gas flow and curtain gas flow, 12 arbitrary units each; Turbo Ionspray gas flow, 6 1/min; source temperature, $375^{\circ} \mathrm{C}$; scan type, multiple reaction monitoring; collision gas, nitrogen; collision gas pressure, 6 arbitrary units. Multiple reaction monitoring conditions for the $[\mathrm{M}+\mathrm{H}]^{+}$ions of both $1^{\prime}$-hydroxymidazolam $(\mathrm{m} / \mathrm{z} 342 \rightarrow 203)$ and $\mathrm{d}_{4}-1^{\prime}$ hydroxymidazolam $(346 \rightarrow 203)$ were as follows: dwell time, $100 \mathrm{~ms}$; declustering potential, $36 \mathrm{~V}$; focusing potential, $170 \mathrm{~V}$; collision energy, $38 \mathrm{eV}$. The following parameters were additionally used: entrance potential, $-10 \mathrm{~V}$; Q1 and Q3 resolution, both unit. Quantification of 1'-hydroxymidazolam was carried out using Analyst (version 1.1; Applied Biosystems). Interday variability in the assay averaged less than $7 \%$.

Pharmacokinetic Analysis. The pharmacokinetics of midazolam and 1'hydroxymidazolam were evaluated by noncompartmental methods using Win-
Nonlin (version 4.1; Pharsight, Mountain View, CA). The terminal elimination rate constant $\left(\lambda_{\mathrm{z}}\right)$ was estimated by linear regression of the terminal portion of the log-transformed concentration versus time curve using at least the last three data points in the terminal elimination phase. The terminal half-life $\left(t_{1 / 2}\right)$ was calculated as $\ln (2) / \lambda_{\mathrm{z}}$. The maximum observed concentration $\left(C_{\max }\right)$ and time to reach $C_{\max }\left(T_{\max }\right)$ were obtained directly from the concentration-time profile. The AUC from time 0 to the last measured concentration $\left(C_{\text {last }}\right)$, $\mathrm{AUC}_{\text {last }}$, was determined using the trapezoidal rule with linear up/log down interpolation. The $\mathrm{AUC}$ from zero to infinite time $\left(\mathrm{AUC}_{0-\infty}\right)$ was calculated as the sum of $\mathrm{AUC}_{\text {last }}$ and the ratio of $C_{\text {last }}$ to $\lambda_{\mathrm{z}}$. The apparent oral clearance of midazolam $(\mathrm{Cl} / \mathrm{F})$ was calculated as the ratio of dose to $\mathrm{AUC}_{0-\infty}$. The metabolite/parent $\mathrm{AUC}$ ratios $\left(\mathrm{AUC}_{\mathrm{m}} / \mathrm{AUC}_{\mathrm{p}}\right)$ were calculated as the ratio of the $\mathrm{AUC}$ for 1'-hydroxymidazolam to the AUC for midazolam.

Statistical Analysis. All the statistical analyses were performed using SigmaStat (version 3.5; Systat Software, Inc., San Jose, CA). The in vitro data are presented as mean \pm S.D. of triplicate determinations. Comparisons between the different brands and concentrations of cranberry juice involving HIMs, between the four cranberry juice E-generated fractions and concentrations involving HIMs, between rCYP3A4 and rCYPA5 with respect to the two concentrations of juice $\mathrm{E}$, and between the juice E-generated fractions and concentrations involving rCYP3A4 and rCYP3A5 were made using a two-way analysis of variance (ANOVA). For the Caco- 2 cell experiment, comparisons between $\mathrm{P}_{\mathrm{app}}$ values were made using a one-way ANOVA. Comparisons between treatment groups at each time point were made using a two-way repeated-measures ANOVA. All the post hoc comparisons were made using Tukey's test when an overall difference resulted $(p<0.05)$.

The sample size for the clinical study $(n=16)$ was calculated based on an $80 \%$ power to detect a $25 \%$ difference in midazolam $\mathrm{AUC}_{0-\infty}$ between cranberry juice and water. Medians and ranges are reported for $T_{\max }$. The Wilcoxon signed-rank test was used to detect a difference in $T_{\max }$. Geometric means and coefficients of variation $(100 \times$ S.D. of the natural log-transformed values $)$ are presented for $C_{\text {max }}, \mathrm{AUC}_{\text {last }}, \mathrm{AUC}_{0-\infty}, \mathrm{Cl} / \mathrm{F}$, terminal $t_{1 / 2}$, and metabolite/parent AUC ratios. Geometric means and $90 \%$ confidence intervals are presented for the ratio of cranberry juice to water with respect to $C_{\max }, \mathrm{AUC}_{\text {last }}, \mathrm{AUC}_{0-\infty}$, $\mathrm{Cl} / \mathrm{F}$, and terminal $t_{1 / 2}$. Paired two-tailed Student's $t$-tests based on $\log$ transformed data were used to detect differences between cranberry juice and water treatments. A $p$ value $<0.05$ was considered statistically significant.

\section{Results}

Inhibitory Effects of Multiple Brands of Cranberry Juice on Enteric CYP3A Activity in Vitro. Whole cranberry juice from five different vendors (A, B, C, D, E) was tested as an inhibitor of midazolam 1'-hydroxylation activity in HIMs. Preliminary experiments had shown that the two concentrations of juice tested $(0.05$ and $0.5 \%$ ) were high enough to discern a differential response between brands, but not so high as to ablate activity. Juices from vendors B, C, $\mathrm{D}$, and $\mathrm{E}$ inhibited activity in a concentration-dependent manner (Fig. 2A). Moreover, the extent of inhibition varied with vendor. At the lower concentration of juice tested, relative to control, the extent of inhibition ranged from $34 \%$ (juice A) to $66 \%$ (juice E). Only juice E differed significantly from the other juices. At the higher concentration of juice tested, the extent of inhibition ranged from $43 \%$ (juice A) to $100 \%$ (juices D and E). Juices C, D, and E differed significantly from juices $\mathrm{A}$ and $\mathrm{B}$, and juice $\mathrm{B}$ differed significantly from juice $\mathrm{A}$. The CYP3A inhibitor ketoconazole $(2 \mu \mathrm{M})$ abolished activity relative to control in this and all the subsequent experiments (data not shown).

Because the cranberry juice from vendor E showed the greatest extent of inhibition in HIM (Fig. 2A), juice E was selected for further characterization. Using natural products chemistry techniques, this juice was extracted/fractionated to generate a hexane-, chloroform-, butanol-, and aqueous-soluble fraction (Fig. 1). To characterize the fraction(s) most concentrated in the CYP3A inhibitory components, each fraction was tested in HIMs. Relative to control, all the fractions inhibited midazolam 1'-hydroxylation in a concentration-dependent manner (Fig. 2B). At the lower concentration tested $(10 \mu \mathrm{g} / \mathrm{ml})$, the 
A

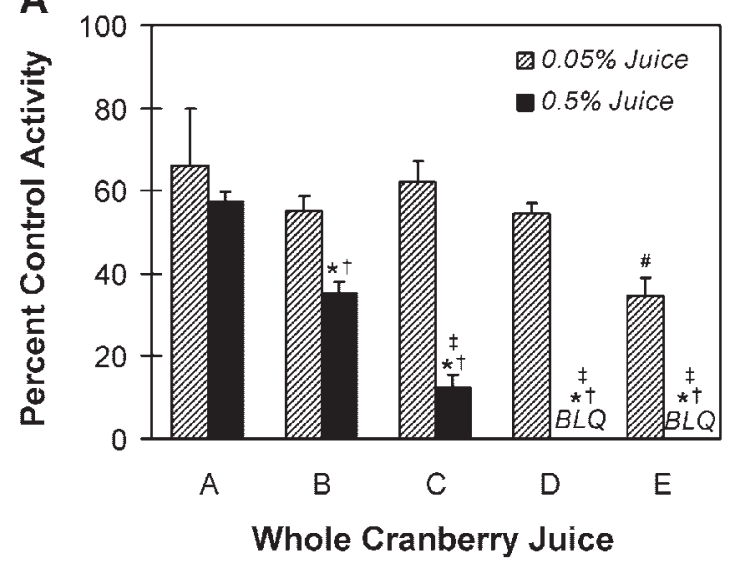

B

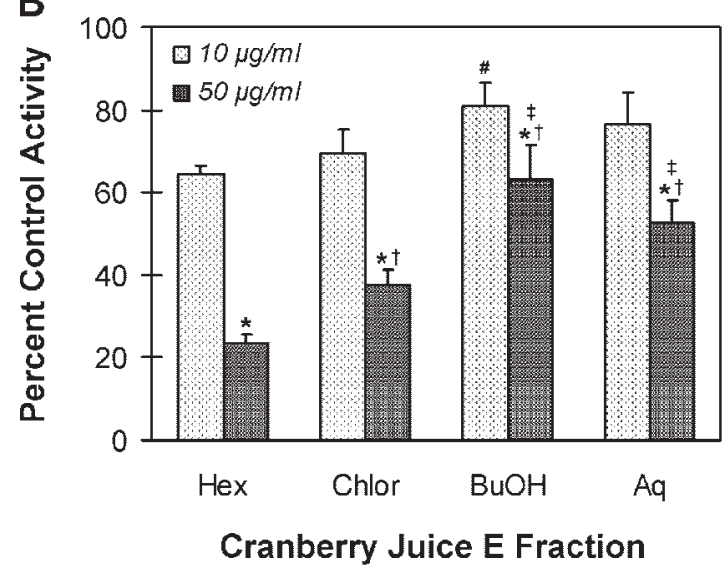

FIG. 2. Inhibitory effects of different brands of commercially available cranberry juices (A) and the various fractions generated from the most potent juice (from vendor E) (B) on the 1'-hydroxylation of midazolam in human intestinal microsomes known not to express CYP3A5. To avoid any perceived conflicts of interest, the identities of the juices were coded as vendors A, B, C, D, and E. Hex, hexane-soluble fraction; Chlor, chloroform-soluble fraction; $\mathrm{BuOH}$, butanol-soluble fraction; Aq, aqueous-soluble fraction; BLQ, below limit of quantification. Bars and error bars denote mean and S.D., respectively, of triplicate incubations. Control activity averaged 87 (A) or 65 (B) $\mathrm{pmol} / \mathrm{min} / \mathrm{mg}$ microsomal protein. *, $p<0.05$ versus $0.05 \%$ corresponding juice (A) or $10 \mu \mathrm{g} / \mathrm{ml}$ corresponding juice fraction (B); $\#, p<0.05$ versus juice A at $0.05 \%$ (A) or hexane-soluble fraction at $10 \mu \mathrm{g} / \mathrm{ml}$ (B); $\dagger, p<0.05$ versus juice A at $0.5 \%$ (A) or hexane-soluble fraction at $50 \mu \mathrm{g} / \mathrm{ml}$ (B); \$, $p<0.05$ versus juice B at $0.5 \%$ (A) or chloroform-soluble fraction at $50 \mu \mathrm{g} / \mathrm{ml}$ (B) (two-way ANOVA, followed by Tukey's test).

extent of inhibition was at most $35 \%$ (hexane-soluble fraction). The hexane-soluble fraction differed significantly from the butanolsoluble fraction. At the higher concentration tested $(50 \mu \mathrm{g} / \mathrm{ml})$, the hexane- and chloroform-soluble fractions were the most potent, inhibiting by 77 and $63 \%$, respectively. Both of these fractions differed significantly from the butanol- and aqueous-soluble fractions, and the hexane-soluble fraction differed significantly from the chloroformsoluble fraction.

The polymorphic CYP3A5 has been reported to be less sensitive than CYP3A4 to inhibition by some therapeutic agents, which could influence the extent of total CYP3A metabolism in some individuals (Isoherranen et al., 2008). As such, the inhibitory effects of juice E and its four derived fractions on midazolam 1'-hydroxylation activity were compared between rCYP3A4 and rCYP3A5. As observed with HIMs, the whole juice inhibited activity in both rCYP3A enzymes in a concentration-dependent manner (Fig. 3). At the lower concentration of juice tested $(0.05 \%)$, rCYP3A5 was inhibited to a lesser extent than rCYP3A4, whereas at a 10-fold higher concentration, activity

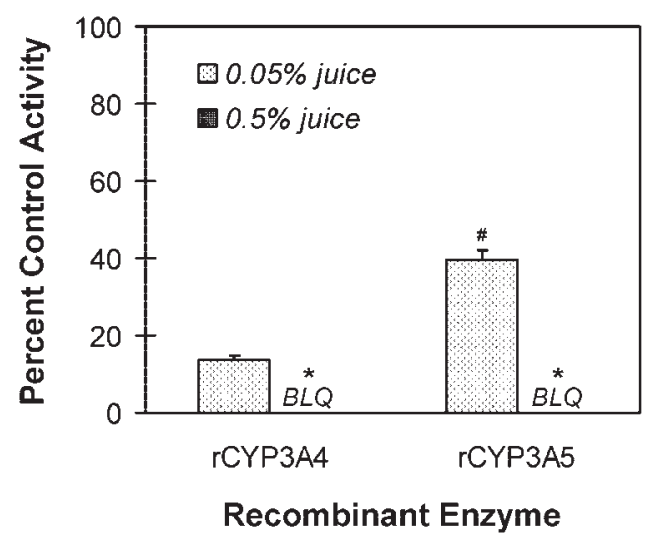

FIG. 3. Inhibitory effects of cranberry juice $\mathrm{E}$ on midazolam 1 '-hydroxylation activity in rCYP3A4 and rCYP3A5 enzymes. Bars and error bars denote mean and S.D., respectively, of triplicate incubations. BLQ, below limit of quantification. Control activity averaged 1.3 (rCYP3A4) or 1.7 (rCYP3A5) pmol/min/pmol. *, $p<$ 0.05 versus $0.05 \%$ juice; $\#, p<0.05$ versus rCYP3A4 at $0.05 \%$ juice (two-way ANOVA, followed by Tukey's test).

was abolished in both enzymes. All the fractions generated from juice $\mathrm{E}$ inhibited both rCYP3A enzymes in a concentration-dependent manner (Fig. 4). At a concentration of $10 \mu \mathrm{g} / \mathrm{ml}$, the extent of inhibition toward rCYP3A4 varied from $10 \%$ (butanol-soluble) to $50 \%$ (hexane-soluble) (Fig. 4A); the extent of inhibition toward rCYP3A5 varied from $0 \%$ (chloroform-soluble) to $23 \%$ (hexanesoluble) (Fig. 4B). For both rCYP3A enzymes, the hexane-soluble fraction differed significantly from all the other fractions. At a concentration of $50 \mu \mathrm{g} / \mathrm{ml}$, the hexane- and chloroform-soluble fractions were the most potent toward $\mathrm{rCYP} 3 \mathrm{~A} 4$, inhibiting activity by at least $75 \%$ (Fig. 4A). Both of these fractions differed significantly from the butanol- and aqueous-soluble fractions, and the hexane-soluble fraction differed significantly from the chloroform-soluble fraction. Only the hexane-soluble fraction showed significant inhibition toward rCYP3A5 (62\%) (Fig. 4B). Only the chloroform-soluble fraction showed an appreciable difference between rCYP3A4 and rCYP3A5, with $\mathrm{rCYP} 3 \mathrm{~A} 4$ being more sensitive to inhibition than rCYP3A5 (75 versus $18 \%$ ).

Effects of Cranberry Juice $E$ on Midazolam Absorption in Caco-2 Cells. The combined physicochemical properties of the clinical test cranberry juice (juice E) and midazolam prompted an evaluation of selected conditions on the absorption of midazolam. First, cranberry juice per se is highly acidic and is sweetened routinely with sugar to improve palatability (Raz et al., 2004); as measured in our laboratory, the $\mathrm{pH}$ of juice $\mathrm{E}$ was 2.5 . Second, at an environmental $\mathrm{pH}$ below 4 , the diazepam ring of midazolam opens, forming a more polar primary amine derivative (Smith et al., 1981). Third, a high glucose concentration has been shown to disrupt the membrane integrity of Caco-2 cell monolayers (D'Souza et al., 2003). Taken together, these observations raised the possibility that sweetened cranberry juice $\mathrm{E}$ could alter the absorption of midazolam in vivo. Accordingly, the potential physicochemical effects of cranberry juice $E$ on the absorptive permeability of midazolam were evaluated using Caco- 2 cell monolayers. The following conditions were tested: acidic $\mathrm{pH}$ (5.5), high glucose concentration (50 g/l, $\mathrm{pH} 7.4)$, and diluted/neutralized cranberry juice (25\% juice, $\mathrm{pH} 7.4$ ).

Under all the conditions, the TEER measurements exceeded 500 $\Omega-\mathrm{cm}^{2}$ and varied $<20 \%$ over the 4 -h experiment, indicating that membrane integrity remained intact with both treatment and time. Under all the treatment conditions, the rate of midazolam appearance in the basolateral compartment was approximately linear up to $1 \mathrm{~h}$ (Fig. 5). The $\mathrm{P}_{\text {app }}$ value under control, acidic, high glucose, and 
A

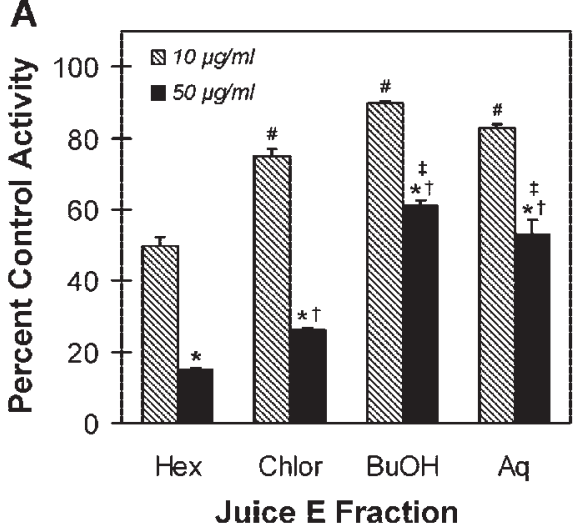

B

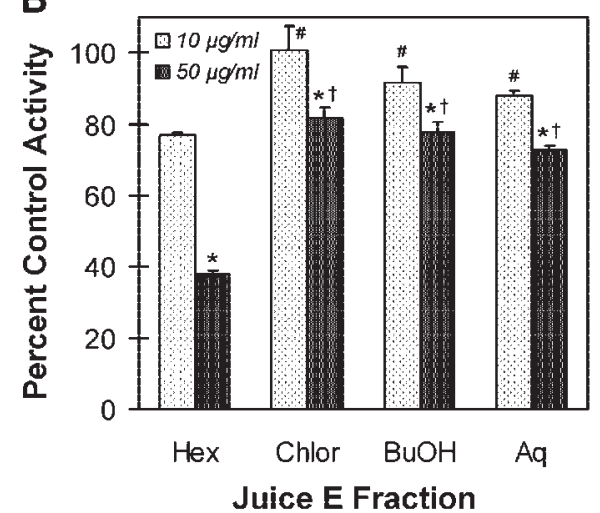

FIG. 4. Inhibitory effects of the various fractions generated from cranberry juice $\mathrm{E}$ on midazolam 1'-hydroxylation activity in rCYP3A4 (A) and rCYP3A5 (B) enzymes. Hex, hexane-soluble fraction; Chlor, chloroform-soluble fraction; $\mathrm{BuOH}$, butanol-soluble fraction; Aq, aqueous-soluble fraction. Bars and error bars denote mean and S.D., respectively, of triplicate incubations. Control activity averaged 1.0 (A) or 1.4 (B) pmol/ $\mathrm{min} / \mathrm{pmol}$ recombinant enzyme. $*, p<0.05$ versus 10 $\mu \mathrm{g} / \mathrm{ml}$ corresponding juice fraction; \#, $p<0.05$ versus hexane-soluble fraction at $10 \mu \mathrm{g} / \mathrm{ml} ; \dagger, p<0.05$ versus hexane-soluble fraction at $50 \mu \mathrm{g} / \mathrm{ml} ; \ddagger, p<0.05$ versus chloroform-soluble fraction at $50 \mu \mathrm{g} / \mathrm{ml}$ (two-way ANOVA, followed by Tukey's test).
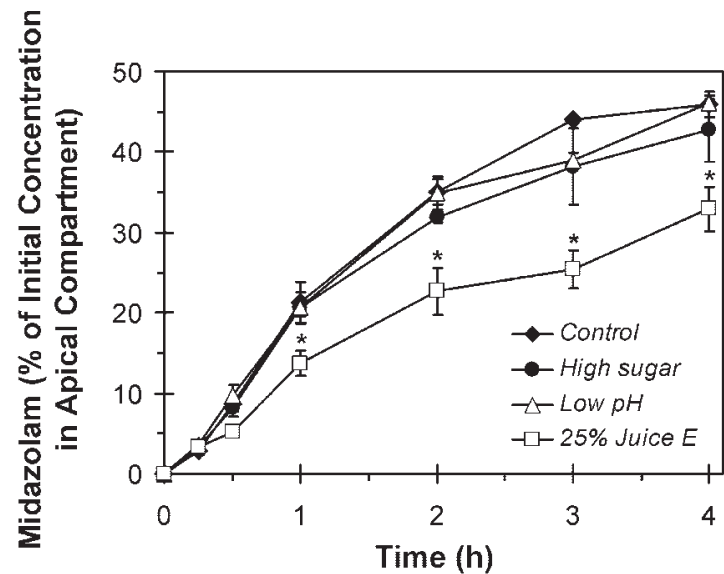

FIG. 5. Effects of high sugar content (50 g/l, $\mathrm{pH} 7.4)$, low $\mathrm{pH}(5.5)$, and $25 \%$ cranberry juice $\mathrm{E}(\mathrm{pH} 7.4)$ on the appearance of midazolam in the basolateral compartment of Caco-2 cell monolayers. Symbols and error bars denote mean and S.D., respectively, of triplicate culture inserts. $*, p<0.05$ versus all the other conditions (two-way ANOVA with repeated measures, followed by Tukey's test).

cranberry juice-supplemented conditions was $19.4 \pm 1.8 \times 10^{-6}$, $21.9 \pm 3.3 \times 10^{-6}, 18.9 \pm 2.8 \times 10^{-6}$, and $12.0 \pm 2.410^{-6} \mathrm{~cm} / \mathrm{s}$, respectively. A significant difference in $\mathrm{P}_{\text {app }}$ was detected between control and cranberry juice treatment and between the acidic and cranberry juice treatments. From 1 h onward, compared with control conditions, only cranberry juice significantly altered midazolam absorption (Fig. 5). The amount of drug appearing in the basolateral compartment was lower in the presence of cranberry juice at all the time points, by 34 to $47 \%$. The total amount of midazolam recovered from the apical and basolateral chambers was $\geq 80 \%$ of the initial amount added to the apical chamber at all the time points and under all the conditions. The amount of midazolam recovered from cell scrapings at the end of the 4-h experiment was similar among all four conditions (7-9\% of the initial amount added to the apical chamber).

Effects of Cranberry Juice $\mathbf{E}$ on Midazolam Pharmacokinetics. The effects of double-strength cranberry juice $\mathrm{E}$ were compared with water on the oral pharmacokinetics of midazolam in 16 healthy participants. Because the causative ingredient(s) underlying a potential midazolam-cranberry juice interaction were/are not known, along with the fact that natural products vary substantially in the composition of bioactive ingredients (Paine and Oberlies, 2007), multiple glasses of double-strength juice were given to maximize the ability to detect an interaction; a similar approach was taken routinely in early drug-grapefruit juice interaction studies (Bailey et al., 1991; Kane and Lipsky, 2000), when the causative ingredients in grapefruit juice were not known. The sweetened double-strength cranberry juice was well tolerated by the majority of the subjects. Most of the subjects reported drowsiness, which is a common side effect of midazolam. Two of the subjects experienced mild nausea, but no vomiting episodes were reported. All of the reported side effects resolved within a few hours.

In general, midazolam was absorbed rapidly when taken with water, with a median time to peak concentration occurring within 30 min (Fig. 6; Table 1). In each of the 16 subjects, compared with water, cranberry juice E slowed the absorption of midazolam, as evidenced by an increase in $T_{\max }$, coupled with a decrease in $C_{\max }$. The increase in $T_{\text {max }}$ varied from 2- to 8-fold, and the decrease in $C_{\text {max }}$ varied from 4 to $70 \%$. Median $T_{\max }$ was increased significantly by 6 -fold, and the geometric mean $C_{\max }$ was decreased significantly by $44 \%$ (Table 1 ). For both phases, the percentage of the $\mathrm{AUC}_{0-\infty}$ extrapolated to infinite time was less than $15 \%$ in the majority of the subjects $(n=14)$ and was at most $24 \%$. Compared with water, cranberry juice $\mathrm{E}$ increased the $\mathrm{AUC}_{\text {last }}$ and $\mathrm{AUC}_{0-\infty}$ in all but four subjects; among all the subjects, the -fold change ranged from 0.9- to 2.6-fold and from 0.9to 2.7-fold, respectively. The geometric mean $\mathrm{AUC}_{\text {last }}$ and $\mathrm{AUC}_{\mathrm{O}-\infty}$ were increased significantly, by approximately $30 \%$, respectively (Fig. 6; Table 1). Correspondingly, in all but four subjects, cranberry juice $\mathrm{E}$ decreased midazolam $\mathrm{Cl} / \mathrm{F}$; among all the subjects, the difference ranged from -63 to $15 \%$ (Fig. 7A). The geometric mean $\mathrm{Cl} / \mathrm{F}$ was decreased significantly by $25 \%$ (Table 1 ). The geometric mean terminal $t_{1 / 2}$ was not significantly different between the two treatments (Table 1).

The formation of 1'-hydroxymidazolam was rapid, regardless of treatment, as exemplified by the simultaneous appearance with midazolam in the systemic circulation (Fig. 6). Accordingly, the median $T_{\max }$ of the metabolite was similar to that of the parent, regardless of treatment ( $p \geq 0.09$ ) (Table 1). Moreover, as with midazolam, cranberry juice slowed the systemic appearance of 1 '-hydroxymidazolam relative to water. Median $T_{\max }$ was increased significantly, by 4 -fold, and the geometric mean $C_{\max }$ was decreased significantly by $68 \%$ (Table 1). For both treatments, the percentage of the $\mathrm{AUC}_{0-\infty}$ extrapolated to infinite time was less than $15 \%$ in most of the subjects $(n=$ 12) and did not exceed $22 \%$. Compared with water, cranberry juice $\mathrm{E}$ decreased the $\mathrm{AUC}_{\text {last }}$ and $\mathrm{AUC}_{\mathrm{O}-\infty}$ of 1'-hydroxymidazolam in all but three subjects; among all the subjects, the difference ranged from approximately -47 to $24 \%$. The geometric mean $\mathrm{AUC}_{\text {last }}$ and $\mathrm{AUC}_{0-\infty}$ were decreased significantly by 20 and $16 \%$, respectively (Fig. 6; Table 1). The systemic elimination of 1'-hydroxymidazolam during the water phase showed formation rate-limited kinetics, as exemplified by the similar geometric mean terminal $t_{1 / 2}$ between midazolam and 1'-hydroxymidazolam ( $p=0.35$ ) (Table 1). Cranberry juice $\mathrm{E}$ appeared not to alter the systemic elimination of the metabolite $(p=0.20)$. Consistent with the effect of juice $\mathrm{E}$ on 


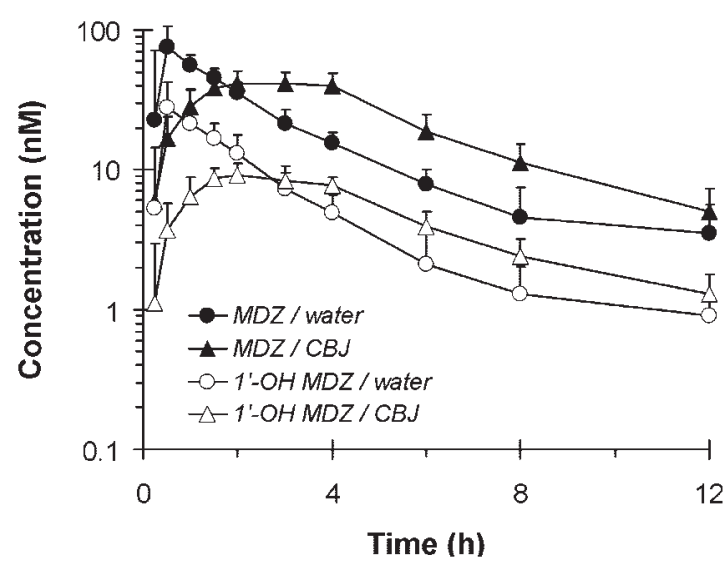

FIG. 6. Geometric mean concentration-time profile of midazolam (MDZ) and 1'hydroxymidazolam (1'-OH MDZ) for 16 healthy volunteers given $3 \times 240-\mathrm{ml}$ glasses of water or sweetened double-strength cranberry juice E (CBJ) and a single oral dose of midazolam $(5 \mathrm{mg})$. Symbols and error bars denote geometric means and upper limits of the $90 \%$ confidence interval, respectively. The 12-h time point for midazolam during the water phase represents the geometric mean of 12 subjects.

midazolam $\mathrm{Cl} / \mathrm{F}$ (Fig. 7A), relative to water, both the partial and total metabolite/parent AUC ratios were decreased in all but three subjects (Fig. 7B); among all the subjects, the difference ranged from -63 to $1 \%$ and from -66 to $1 \%$, respectively. The geometric means of the partial and total metabolite/parent AUC ratios were decreased significantly by $\sim 40 \%$ (Table 1 ).

\section{Discussion}

Based on the inconsistencies in the literature regarding whether cranberry juice can elicit clinically relevant interactions with certain medications, coupled with the inherent difficulties that can arise when evaluating the drug interaction liability of a natural product, the current work was undertaken to address three objectives. The first was to determine whether cranberry juice has any interaction liability with drugs that undergo extensive first-pass metabolism by enteric CYP3A. As aforementioned, it remains unclear whether the in vivo inconsistencies reflect species differences, study design/sample size, and/or variability of the natural product study materials. The second objective was to show that natural product study materials, all seemingly of the same content, can act distinctly from each other, both chemically and biologically. A drug-natural product interaction study in the universal sense is impossible because natural products vary considerably based on a host of factors, including climate, growing season, and processing procedures (Paine and Oberlies, 2007). Accordingly, it seems premature to draw broad conclusions from an investigation of one brand of the natural product. The third objective was to develop a more rigorous in vitro-in vivo approach that can be used prospectively to evaluate the drug interaction liability of a dietary substance. That is, if current models are inappropriate, how can we better investigate the drug interaction potential of a dietary component? Do we wait for a serendipitous observation, as with grapefruit juice (Bailey et al., 1991), or do we examine one brand chosen randomly, as apparently occurred in recent studies? To address this issue, well established techniques used to characterize drug disposition were combined with those used to characterize natural products (Paine and Oberlies, 2007).

The inhibitory effects of different brands of cranberry juice were evaluated first on enteric CYP3A-mediated metabolism using a relevant human-derived in vitro system and an established probe substrate that can be given to humans. Consistent with the general interbrand variation in natural products regarding the composition of bioactive components, the extent of inhibition varied considerably among just five brands tested, ranging from $34 \%$ to abolishment. Such interbrand variability is undoubtedly much greater in the broader marketplace. If such a scenario were to occur with five different lots of a drug, a single lot would not be considered representative of all the others. Likewise, a single brand/lot of cranberry juice would not be representative of the entire marketplace.

The most potent of the tested juices (E) was selected for further characterization, which was to fractionate the juice to produce a hexane-, chloroform-, butanol-, and aqueous-soluble fraction and to evaluate the effects of each on enteric CYP3A activity. The hexaneand chloroform-soluble fractions showed the greatest extent of inhibition in both HIMs and rCYP3A4 and were concentration-dependent, indicating that the CYP3A inhibitors in juice E resided largely in these two more lipophilic fractions. As has been reported for several therapeutic agents, including ketoconazole, fluconazole, diltiazem, and mifepristone (Isoherranen et al., 2008), the whole juice (at the lower concentration), as well as the chloroform-soluble fraction (at the higher concentration), showed greater inhibition toward rCYP3A4 than rCYP3A5. Further purification of the hexane- and chloroformsoluble fractions is ongoing to identify the inhibitory compound(s), which will enable the determination of whether juice E, and cranberry juice in general, contains a selective CYP3A4 inhibitor.

Potential physicochemical effects of cranberry juice E were evaluated next on the absorptive permeability of midazolam in Caco-2 cells, a human intestinal cell line used routinely as a model of the small intestinal epithelium (Press and Di Grandi, 2008). Compared with control conditions, only juice $\mathrm{E}$ altered midazolam absorption significantly. As reflected in the $\mathrm{P}_{\text {app }}$ values, juice $\mathrm{E}$ ( $\mathrm{pH}$ adjusted to 7.4), but not high sugar content or low $\mathrm{pH}$ conditions, slowed midazolam appearance in the basolateral compartment. From $1 \mathrm{~h}$ onward, midazolam concentration in the basolateral compartment was always lower than that under all the other conditions by at least $34 \%$. The similar total recoveries of midazolam from the apical and basolateral compartments at all the time points $(\geq 80 \%)$, as well as from the cell scrapings at the end of the 4 -h experiment $(\sim 8 \%)$, under all the conditions indicated that these observations were not artifacts of the HPLC/MS/MS method.

As proof of concept, the effects of cranberry juice E were compared with water on the oral pharmacokinetics of midazolam in healthy volunteers. Unlike the two clinical studies that showed no interaction between a different cranberry juice product and cyclosporine (Grenier et al., 2006) or midazolam (Lilja et al., 2007), but consistent with current observations from Caco-2 cells, juice E decreased the rate of midazolam absorption, as evidenced by a 6 -fold increase in $T_{\max }$ and $\sim 40 \%$ decrease in $C_{\max }$. The prolonged $T_{\max }$ suggested a decreased gastric emptying rate, which may have been associated with the high sugar content of the juice. The effects of the acidic $\mathrm{pH}$ of the juice and/or stomach on the physicochemical properties of midazolam (i.e., ring-opening of the diazepam ring) could also have contributed to the prolonged $T_{\max }$ and decreased $C_{\max }$. However, if such an event occurred, it seemed to be transient, as ultimately, an increase, rather than decrease, in midazolam systemic exposure (AUC) resulted. The lack of an effect of high sugar content and low $\mathrm{pH}$ conditions on midazolam absorptive permeability in Caco- 2 cells further suggested that components inherent to cranberry juice were responsible for the slowed absorption.

Although cranberry juice $\mathrm{E}$ decreased the rate of midazolam absorption, systemic exposure was enhanced significantly, as exemplified by an $\sim 30 \%$ increase in AUC. The lack of effect on terminal half-life suggested that the juice altered primarily intestinal, but not hepatic, processes. An increase in the fraction of the midazolam dose 
TABLE 1

Pharmacokinetics of midazolam and 1'-hydroxymidazolam in 16 healthy volunteers given $3 \times 240$-ml glasses of water or sweetened double-strength cranberry juice from vendor $E(C B J)$ and a single oral dose of midazolam $(5 \mathrm{mg})$

\begin{tabular}{|c|c|c|c|c|}
\hline \multirow{2}{*}{ Measure $^{a}$} & \multicolumn{2}{|c|}{ Geometric Mean } & \multirow{2}{*}{ CBJ/Water Ratio } & \multirow{2}{*}{$p$ Value $^{b}$} \\
\hline & Water & CBJ & & \\
\hline & $C V \%$ & $\mathrm{CV} \%$ & $90 \% C I$ & \\
\hline \multicolumn{5}{|l|}{ Midazolam } \\
\hline$T_{\max }(\mathrm{h})$ [median (range)] & $0.5(0.25-1.5)$ & $3.0(1.0-4.0)$ & & $<0.001$ \\
\hline$C_{\max }(\mathrm{nM})$ & $88(44)$ & $50(35)$ & $0.56[0.49-0.64]$ & $<0.001$ \\
\hline $\mathrm{AUC}_{\text {last }}(\mathrm{nM} \cdot \mathrm{h})$ & $197(30)$ & $259(31)$ & $1.31[1.18-1.46]$ & $<0.001$ \\
\hline $\operatorname{AUC}_{0-\infty}(\mathrm{nM} \cdot \mathrm{h})$ & $215(33)$ & $286(30)$ & $1.33[1.17-1.50]$ & 0.001 \\
\hline $\mathrm{Cl} / \mathrm{F}(1 / \mathrm{h})$ & $71(33)$ & $54(30)$ & $0.75[0.67-0.85]$ & 0.001 \\
\hline$t_{1 / 2}(\mathrm{~h})$ & $2.9(41)$ & $2.9(25)$ & $1.01[0.86-1.19]$ & 0.93 \\
\hline \multicolumn{5}{|l|}{ 1'-Hydroxymidazolam } \\
\hline$T_{\max }(\mathrm{h})[$ median (range) $]$ & $0.5(0.25-1.5)$ & $2.0(1.0-6.0)$ & & $<0.001$ \\
\hline$C_{\max }(\mathrm{nM})$ & $33(54)$ & $10(29)$ & $0.32[0.27-0.38]$ & $<0.001$ \\
\hline $\mathrm{AUC}_{\text {last }}(\mathrm{nM} \cdot \mathrm{h})$ & $69(35)$ & $55(23)$ & $0.80[0.73-0.88]$ & 0.002 \\
\hline $\operatorname{AUC}_{0-\infty}(\mathrm{nM} \cdot \mathrm{h})$ & $74(37)$ & $62(25)$ & $0.84[0.77-0.92]$ & 0.005 \\
\hline$t_{1 / 2}(\mathrm{~h})$ & $3.2(35)$ & $3.3(19)$ & $1.04[0.89-1.20]$ & 0.68 \\
\hline \multicolumn{5}{|l|}{ Metabolite/parent AUC ratios } \\
\hline$\left(\mathrm{AUC}_{\mathrm{m}} / \mathrm{AUC} \mathrm{C}_{\mathrm{p}}\right)_{\text {last }}$ & $0.34(49)$ & $0.21(37)$ & & $<0.001$ \\
\hline$\left(\mathrm{AUC}_{\mathrm{m}} / \mathrm{AUC}_{\mathrm{p}}\right)_{0-\infty}$ & $0.35(50)$ & $0.22(39)$ & & $<0.001$ \\
\hline
\end{tabular}

${ }^{a} T_{\max }$, time to reach maximum concentration $\left(C_{\max }\right) ; \mathrm{AUC}_{\text {last }}$, area under the curve from time 0 to the last measurable concentration; $\mathrm{AUC}$ -, area under the curve from 0 to infinite $\mathrm{h} ; \mathrm{Cl} / \mathrm{F}$, apparent oral clearance; $t_{1 / 2}$, terminal half-life

${ }^{b}$ Comparisons between water and CBJ for $T_{\max }$ were made using the Wilcoxon signed-rank test; comparisons between water and CBJ for all the other measures were made using the paired Student's $t$-test.
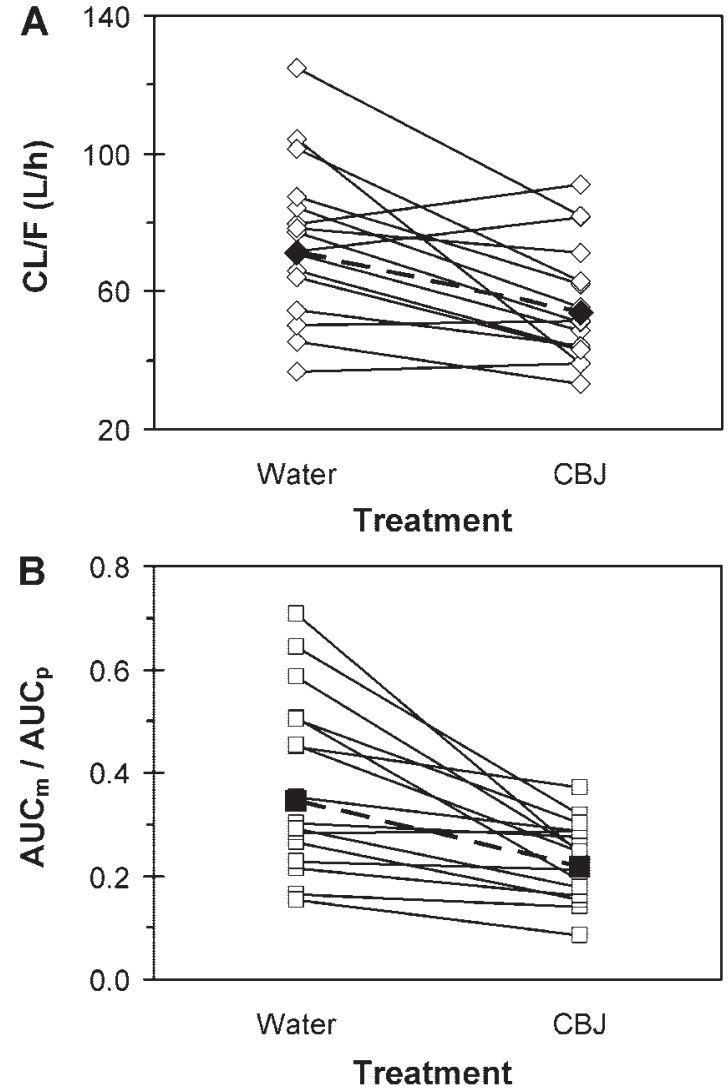

FIG. 7. Effects of $3 \times 240$-ml glasses of water and sweetened double-strength cranberry juice $\mathrm{E}(\mathrm{CBJ})$ on the oral clearance $(\mathrm{Cl} / \mathrm{F})(\mathrm{A})$ of midazolam and the $1^{\prime}$-hydroxymidazolam/midazolam AUC ratio $\left.\left[\left(\mathrm{AUC}_{\mathrm{m}} / \mathrm{AUC}_{\mathrm{p}}\right)_{\left.0_{-\infty}\right]}\right] \mathrm{B}\right)$ in each of 16 healthy volunteers given a single oral dose of midazolam $(5 \mathrm{mg})$. Open symbols and solid lines denote individual values. Filled symbols and dashed lines denote geometric mean values.

absorbed into the intestinal wall by juice $\mathrm{E}$ was unlikely because when midazolam is given orally alone, the urinary recovery of midazolam and metabolites is similar to that after an intravenous dose, indicating that midazolam is absorbed completely into the intestinal wall (Heizmann et al., 1983; Thummel et al., 1996; Gorski et al., 1998). Moreover, because midazolam was given as a syrup under fasted conditions and exhibited a $\mathrm{P}_{\mathrm{app}}\left(>10 \times 10^{-6} \mathrm{~cm} / \mathrm{s}\right)$ characteristic of a highly permeable drug (Press and Di Grandi, 2008), regardless of the presence of juice E, it seems unlikely that the juice shifted the absorption of midazolam to more distal regions of the small intestine, where CYP3A activity is lower than in more proximal regions (Paine et al., 1997). Collectively, the enhanced AUC by juice E was probably the result of inhibition of intestinal first-pass metabolism. The decrease in the 1 '-hydroxymidazolam/midazolam AUC ratio, resulting from both an increase in midazolam AUC and decrease in metabolite AUC, coupled with the observation that 1'-hydroxymidazolam tracked closely with midazolam (i.e., displayed formation rate-limited kinetics) further support this contention. Inhibition of CYP3A-mediated metabolism in the intestine rather than the liver concurs with the in vivo study in rats (Uesawa and Mohri, 2006), arguing against species differences as an explanation for the inconsistencies observed in the literature.

The midazolam-cranberry juice interaction shown in the current work may have a 2-fold clinical impact. First, the slowed absorption may occur with other benzodiazepine receptor agonists. Short- and intermediate-acting agents indicated for insomnia (e.g., triazolam, zolpidem, zaleplon, eszopiclone) achieve $C_{\max }$ and onset of action within 0.5 to $2 \mathrm{~h}$ (Morin et al., 2007). Thus, a slowed absorption may in turn slow the onset of sedation of drugs with effects dependent on $C_{\max }$. Furthermore, if the mechanism is nonspecific, the scope of the interaction may encompass other oral agents whose effects depend on a timely onset, such as those indicated for pain and migraine. A second clinical implication is that inhibition of intestinal first-pass midazolam metabolism by cranberry juice may extend to other oral CYP3A substrates. Although the average increase in midazolam AUC was relatively modest, interindividual variability was evident among just 16 subjects, ranging from negligible to 2.7 -fold. These outcomes are comparable with the effects of grapefruit juice on midazolam AUC (Kupferschmidt et al., 1995; Farkas et al., 2007). Taken together, an enhanced drug systemic exposure elicited by certain brands of cranberry juice may increase the risk for side effects of more sensitive CYP3A 
substrates, including some HMG CoA reductase inhibitors (e.g., lovastatin, simvastatin) and cardiovascular agents (e.g., felodipine, amiodarone), as well as substrates with a narrow therapeutic window (e.g., cyclosporine, tacrolimus) (Farkas and Greenblatt, 2008). Moreover, because of the extensive substrate overlap between CYP3A and P-gp, the magnitude of the effect may also depend on the interplay between CYP3A and P-gp, and/or some other transporter, in a given individual (Paine and Oberlies, 2007). Furthermore, cranberry juice is consumed widely by women and elderly persons (Jepson and Craig, 2008). Because these populations also have a high rate of drug and natural product supplement usage (Leibovitch et al., 2004; Schwartz, 2007), a large segment of the overall population could be at risk for certain drugcranberry juice interactions. Finally, cranberry juice is often taken chronically, sometimes at the earliest onset of UTI symptoms. Whether the effects of long-term cranberry juice exposure on drug disposition are more pronounced than acute exposure merits further investigation.

In summary, a systematic approach, which began with in vitro testing and culminated with a proof-of-concept clinical study, enabled the identification of a cranberry juice product that elicited a pharmacokinetic interaction with an established CYP3A probe substrate. This approach limited the need for conducting more time-consuming and costly clinical studies to compare the effects of different brands of juice. Moreover, unlike the production of drug products, the profile of secondary metabolites in natural products varies substantially. That is, whereas a pharmaceutical agent has a defined molecular formula and chemical structure, regardless of the manufacturer, there are hundreds of constituents in "cranberry juice," and the profile of such constituents can vary considerably between growing conditions, formulations, brands, and even lots within the same brand. The scientific literature is replete with claims, both positive and negative, about herbal products and other dietary substances, typically referring to such products in a general sense (e.g., cranberry, St. John's wort). The current work substantiates that rigorous in vitro characterization of dietary substances should be undertaken before initiation of clinical drug-diet interaction studies.

Acknowledgments. We thank Nicole White (University of North Carolina Eshelman School of Pharmacy) for technical assistance with the analysis of human plasma for midazolam; Michael Gardner and Dr. Jonathon Bundy (Mass Spectrometry Research Group, Research Triangle Institute) for consultation on the analysis of human plasma for 1'-hydroxymidazolam; and Dr. Adam Persky (University of North Carolina Eshelman School of Pharmacy) for consultation with the pharmacokinetic analysis. M.F.P. dedicates this article to Dr. David P. Paine.

\section{References}

Avorn J, Monane M, Gurwitz JH, Glynn RJ, Choodnovskiy I, and Lipsitz LA (1994) Reduction of bacteriuria and pyuria after ingestion of cranberry juice. JAMA 271:751-754.

Bailey DG, Spence JD, Munoz C, and Arnold JM (1991) Interaction of citrus juices with felodipine and nifedipine [see comments]. Lancet 337:268-269.

Cain CC, Lee D, Waldo RH 3rd, Henry AT, Casida EJ Jr, Wani MC, Wall ME, Oberlies NH, and Falkinham JO 3rd (2003) Synergistic antimicrobial activity of metabolites produced by a nonobligate bacterial predator. Antimicrob Agents Chemother 47:2113-2117.

D'Souza VM, Shertzer HG, Menon AG, and Pauletti GM (2003) High glucose concentration in isotonic media alters caco-2 cell permeability. AAPS Pharm Sci 5:E24.

Farkas D and Greenblatt DJ (2008) Influence of fruit juices on drug disposition: discrepancies between in vitro and clinical studies. Expert Opin Drug Metab Toxicol 4:381-393.

Farkas D, Oleson LE, Zhao Y, Harmatz JS, Zinny MA, Court MH, and Greenblatt DJ (2007) Pomegranate juice does not impair clearance of oral or intravenous midazolam, a probe for cytochrome P450-3A activity: comparison with grapefruit juice. J Clin Pharmacol 47:286294.

Foxman B (2003) Epidemiology of urinary tract infections: incidence, morbidity, and economic costs. Dis Mon 49:53-70.
Gorski JC, Jones DR, Haehner-Daniels BD, Hamman MA, O'Mara EM Jr, and Hall SD (1998) The contribution of intestinal and hepatic CYP3A to the interaction between midazolam and clarithromycin. Clin Pharmacol Ther 64:133-143.

Grenier J, Fradette C, Morelli G, Merritt GJ, Vranderick M, and Ducharme MP (2006) Pomelo juice, but not cranberry juice, affects the pharmacokinetics of cyclosporine in humans. Clin Pharmacol Ther 79:255-262.

Gupta K, Chou MY, Howell A, Wobbe C, Grady R, and Stapleton AE (2007) Cranberry products inhibit adherence of p-fimbriated Escherichia coli to primary cultured bladder and vaginal epithelial cells. J Urol 177:2357-2360.

Heizmann P, Eckert M, and Ziegler WH (1983) Pharmacokinetics and bioavailability of midazolam in man. Br J Clin Pharmacol 16:43S-49S.

Howell AB and Foxman B (2002) Cranberry juice and adhesion of antibiotic-resistant uropathogens. JAMA 287:3082-3083.

Isoherranen N, Ludington SR, Givens RC, Lamba JK, Pusek SN, Dees EC, Blough DK, Iwanaga K, Hawke RL, Schuetz EG, et al. (2008) The influence of CYP3A5 expression on the exten of hepatic CYP3A inhibition is substrate-dependent: an in vitro-in vivo evaluation. Drug Metab Dispos 36:146-154.

Jepson RG and Craig JC (2008) Cranberries for preventing urinary tract infections. Cochrane Database Syst Rev (1): CD001321

Kanazawa H, Okada A, Igarashi E, Higaki M, Miyabe T, Sano T, and Nishimura R (2004) Determination of midazolam and its metabolite as a probe for cytochrome P450 3A4 phenotype by liquid chromatography-mass spectrometry. J Chromatogr A 1031:213-218.

Kane GC and Lipsky JJ (2000) Drug-grapefruit juice interactions. Mayo Clin Proc 75:933-942. Kinghorn AD, Farnsworth NR, Soejarto DD, Cordell GA, Swanson SM, Pezzuto JM, Wani MC, Wall ME, Oberlies NH, Kroll DJ, et al. (2003) Novel strategies for the discovery of plant-derived anticancer agents. Pharm Biol 41:53-67.

Kupferschmidt HH, Ha HR, Ziegler WH, Meier PJ, and Krähenbühl S (1995) Interaction between grapefruit juice and midazolam in humans. Clin Pharmacol Ther 58:20-28.

Leibovitch ER, Deamer RL, and Sanderson LA (2004) Food-drug interactions: careful drug selection and patient counseling can reduce the risk in older patients. Geriatrics 59:19-22.

Lilja JJ, Backman JT, and Neuvonen PJ (2007) Effects of daily ingestion of cranberry juice on the pharmacokinetics of warfarin, tizanidine, and midazolam-probes of CYP2C9, CYP1A2, and CYP3A4. Clin Pharmacol Ther 81:833-839.

Liu Y, Black MA, Caron L, and Camesano TA (2006) Role of cranberry juice on molecular-scale surface characteristics and adhesion behavior of Escherichia coli. Biotechnol Bioeng 93:297305.

Lynch DM (2004) Cranberry for prevention of urinary tract infections. Am Fam Physician 70:2175-2177.

McMurdo ME, Bissett LY, Price RJ, Phillips G, and Crombie IK (2005) Does ingestion of cranberry juice reduce symptomatic urinary tract infections in older people in hospital? A double-blind, placebo-controlled trial. Age Ageing 34:256-261.

Morin AK, Jarvis CI, and Lynch AM (2007) Therapeutic options for sleep-maintenance and sleep-onset insomnia. Pharmacotherapy 27:89-110.

Mouly SJ, Paine MF, and Watkins PB (2004) Contributions of CYP3A4, P-glycoprotein, and serum protein binding to the intestinal first-pass extraction of saquinavir. J Pharmacol Exp Ther 308:941-948.

Paine MF, Hart HL, Ludington SS, Haining RL, Rettie AE, and Zeldin DC (2006) The human intestinal cytochrome P450 "pie." Drug Metab Dispos 34:880-886.

Paine MF, Khalighi M, Fisher JM, Shen DD, Kunze KL, Marsh CL, Perkins JD, and Thummel KE (1997) Characterization of interintestinal and intraintestinal variations in human CYP3Adependent metabolism. J Pharmacol Exp Ther 283:1552-1562.

Paine MF and Oberlies NH (2007) Clinical relevance of the small intestine as an organ of drug elimination: drug-fruit juice interactions. Expert Opin Drug Metab Toxicol 3:67-80.

Phifer SS, Lee D, Seo EK, Kim NC, Graf TN, Kroll DJ, Navarro HA, Izydore RA, Jiménez F, Garcia R, et al. (2007) Alvaradoins E-N, antitumor and cytotoxic anthracenone C-glycosides from the leaves of Alvaradoa haitiensis. J Nat Prod 70:954-961.

Press B and Di Grandi D (2008) Permeability for intestinal absorption: Caco-2 assay and related issues. Curr Drug Metab 9:893-900.

Raz R, Chazan B, and Dan M (2004) Cranberry juice and urinary tract infection. Clin Infect Dis 38:1413-1419.

Schmiedlin-Ren P, Thummel KE, Fisher JM, Paine MF, Lown KS, and Watkins PB (1997) Expression of enzymatically active CYP3A4 by Caco-2 cells grown on extracellular matrixcoated permeable supports in the presence of $1 \alpha, 25$-dihydroxyvitamin $\mathrm{D}_{3}$. Mol Pharmacol 51:741-754.

Schwartz JB (2007) The current state of knowledge on age, sex, and their interactions on clinical pharmacology. Clin Pharmacol Ther 82:87-96.

Smith MT, Eadie MJ, and Brophy TO (1981) The pharmacokinetics of midazolam in man. Eur J Clin Pharmacol 19:271-278.

Stothers L (2002) A randomized trial to evaluate effectiveness and cost effectiveness of naturopathic cranberry products as prophylaxis against urinary tract infection in women. Can J Urol 9:1558-1562.

Thummel KE, O'Shea D, Paine MF, Shen DD, Kunze KL, Perkins JD, and Wilkinson GR (1996) Oral first-pass elimination of midazolam involves both gastrointestinal and hepatic CYP3Amediated metabolism. Clin Pharmacol Ther 59:491-502.

Uesawa Y and Mohri K (2006) Effects of cranberry juice on nifedipine pharmacokinetics in rats. J Pharm Pharmacol 58:1067-1072.

Wang MZ, Wu JQ, Bridges AS, Zeldin DC, Kornbluth S, Tidwell RR, Hall JE, and Paine MF (2007) Human enteric microsomal CYP4F enzymes $O$-demethylate the antiparasitic prodrug pafuramidine. Drug Metab Dispos 35:2067-2075.

Address correspondence to: Mary F. Paine, 2320 Kerr Hall, CB \#7360, Eshelman School of Pharmacy, University of North Carolina, Chapel Hill, NC 27599-7360. E-mail: mpaine@unc.edu 